
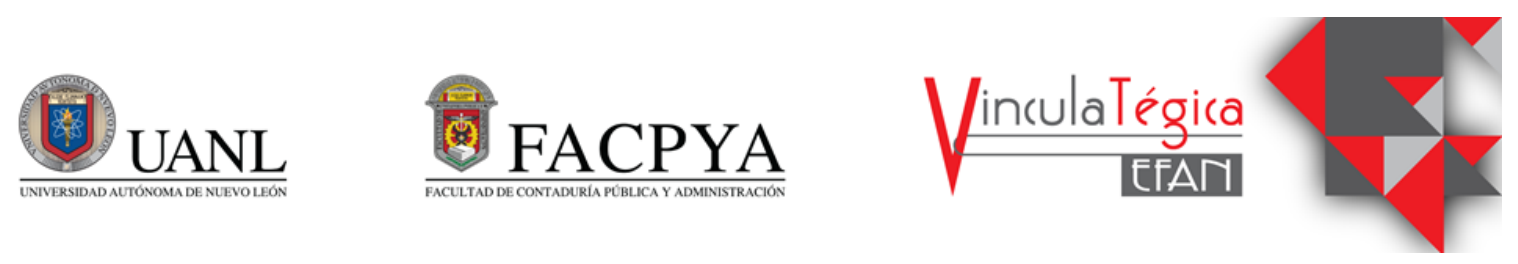

\title{
Un análisis de la percepción sobre la gentrificación inmobiliaria en Monterrey (Nuevo León)
}

\author{
Laura Martínez Cazares ${ }^{1}$ y Efraín Romero Jasso ${ }^{2}$ \\ ${ }^{1}$ Estudiante de posgrado de la maestría en valuación de la facultad de arquitectura de la Universidad Autónoma de Nuevo \\ León, laura.martinezcz@uanl.edu.mx,Av Av.Universidad s/n San Nicolás de los Garza, Nuevo León C.P. 66455 \\ ${ }^{2}$ Profesor e investigador de la facultad de arquitectura en la Universidad Autónoma de Nuevo León, \\ romerojasso@gmail.com, Av. Universidad s/n San Nicolás de los Garza, Nuevo León C.P. 66455 Tel. (81) 8329-4000.
}

Información del artículo revisado por pares

Fecha de aceptación: junio-2021

Fecha de publicación en línea: diciembre-2021

DOI: https://doi.org/10.29105/vtga7.1-121

\section{Resumen}

El desarrollo urbano que ha tenido el área metropolitana de Monterrey (AMM) ha generado el crecimiento de la mancha urbana y la gentrificación de la periferia de la ciudad. El objetivo en esta investigación es conocer las características de los pobladores de la zona sur de la ciudad de Monterrey en lo que respecta a sus componentes sociales y urbanos. Donde la división social es muy notoria a causa de los nuevos desarrollos inmobiliarios y el estrato socioeconómico de los nuevos habitantes de los asentamientos ya existentes. Se utilizó y aplico una encuesta cuantitativa por conveniencia para demostrar si este crecimiento es para el beneficio de la población de la segregación socio-espacial, la percepción de la población por la transformación de su entorno, la densificación del suelo, la comparación del marco económico y territorial para llegar a una conclusión aterrizada sobre este tema y conocer si la llegada de estos desarrollos beneficia a la mayor parte de la población o sólo a la parte más privilegiada.

Palabras clave: Densificación, desarrollo urbano, gentrificación, segregación, invasión.

\section{INTRODUCCIÓN}

El objetivo de esta investigación es conocer las características de los pobladores de la zona sur de la ciudad de Monterrey Nuevo León en lo que respecta a sus componentes sociales y urbanos. Se definen y estudian criterios como el perfil socioeconómico, la densidad habitacional, antigüedad de residencia,

\begin{abstract}
The urban development of the Monterrey metropolitan area (MMA) has generated the growth of the urban sprawl and the gentrification of the city's periphery. The objective of this research is to know the traits of the residents of the southern area of the city of Monterrey in terms of its social and urban components. Where the social division is very noticeable due to the new real estate developments and the socioeconomic stratum of the new residents of the existing settlements. A quantitative survey of convenience was used and applied to demonstrate if this growth is for the benefit of the population of the socio-spatial segregation, the perception of the population by the transformation of their environment, the densification of the soil, the comparison of the economic and territorial framework to reach a grounded conclusion on this issue and to know if the arrival of these developments benefits the majority of the population or only the most privileged part.
\end{abstract}

Keywords: densification, urban development, Gentrification, segregation, invation.

JEL: J11, J16.

demografía y seguridad. Estos factores son útiles para determinar la presencia de la segregación social y espacial. Para esto se hizo una revisión de los aspectos históricos de la zona con un antes y un después de la llegada de los desarrollos inmobiliarios (2000) para conocer si estos han beneficiado en cuanto a calidad de vida, accesibilidad en bienes 
materiales, seguridad y plusvalía o afectado a la población en cuanto a segregación, aumento en servicios y complicación en la movilidad. Se sabe que Nuevo León es conocido por el rápido crecimiento urbano y que este mismo ha invadido las periferias desorganizando el uso de suelo y la función espacial de cada zona. (IMPLAN 20132025).

La gentrificación es un proceso de rehabilitación urbana y social de una zona deprimida o deteriorada, que provoca un desplazamiento de los antiguos habitantes del área al sentirse invadidos por otros habitantes de un nivel social más alto (Díaz,2013).

La aplicación de este término en los estudios urbanos ha derivado un gran debate en el último tiempo, de acuerdo con Mendoza (2016) centrado fundamentalmente en la pertinencia y adecuación de este concepto a las transformaciones que experimenta la ciudad actual.

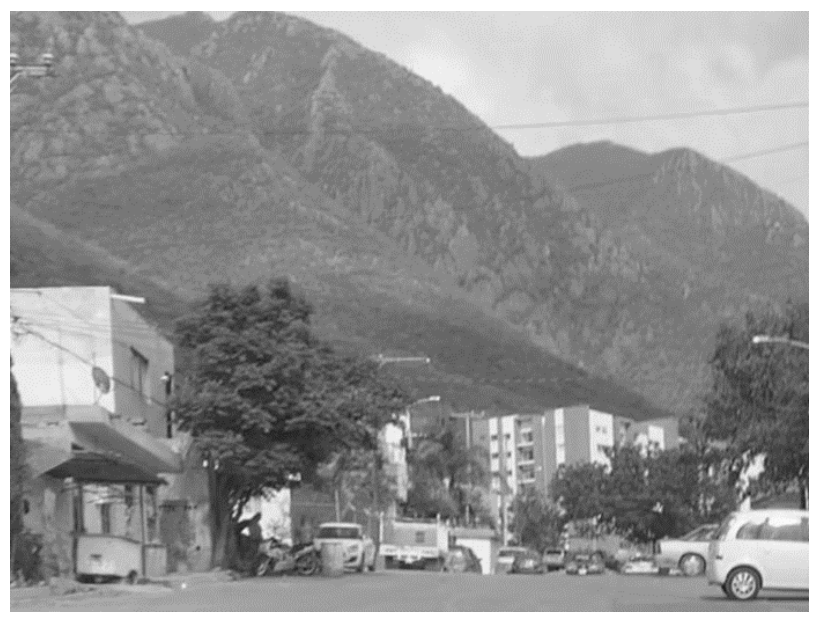

Figura 1: Contraposición de barrio existente contra desarrollo inmobiliario "torre Gaia". Fuente: propia.

La participación activa del mercado inmobiliario y su crecimiento desmedido ha causado invasión a los barrios de la periferia afectando sus usos, costumbres por la diferencia de clases sociales.

Esto es causado por la desigualdad en ingresos económicos e igualdad de oportunidades. (Clark 2005) (Figura 1).

\section{MARCO TEÓRICO}

La fundación de Monterrey (1596) tiene como antecedentes las exploraciones colonizadoras al noreste de la Nueva España, entonces. - Monterrey tenía 11,044 habitantes en su casco urbano y ranchos aledaños. En 1829 elevó su población a 20,000 habitantes, sostenidos principalmente por las actividades agrícolas y ganaderas. En 1854 se estableció la primera gran empresa fabril en el ramo textil "La Fama". Hacia 1870 alcanzó los 33,811 habitantes y 35,386 en 1880 y, tres años más tarde se fundó la Cámara de Comercio de Monterrey, época en que la actividad empresarial y los capitales existentes empezaron a canalizarse hacia la industria. Para 1940 Monterrey registró un crecimiento poblacional notable alcanzando a tener 200,000 residentes, lo que obligó a acelerar la construcción de casas habitación de todas las categorías. Monterrey diversificó considerablemente su estructura económica alcanzó niveles de industrialización avanzados, entre 1960-1980 (Alanís, Glafiro, Cervantes, 1995).

El proceso de urbanización en México característico de la segunda mitad del siglo XX ha producido concentraciones urbanas en algunas zonas del país que han desbordado las divisiones político administrativas municipales para constituirse en metrópolis, tal es el caso de la Zona Conurbada de Monterrey. El proceso de expansión territorial y poblacional del Área Metropolitana de Monterrey, se inició en la década de los cuarenta como consecuencia del proceso de urbanizaciónindustrialización que experimentó el país en esos años. Dentro de ese proceso, Monterrey se distinguió como uno de los principales polos de atracción demográfica. Entre 1940 y el año 2010, la población se incrementó 17 veces y el Área Urbana se extendió a nueve municipios (Barbarín, 1995).

\subsection{La gentrificación de la ciudad}

Como se mencionó, el fenómeno de transformación urbana en donde los barrios populares/decadentes, son invadidos por inversionistas privados o en casos muy específicos, públicos, genera movilizaciones de los residentes tradicionales, puesto que resulta más costosa la vida en un sector que ahora está revalorizado, y son desplazados por nuevos grupos sociales con mayor poder adquisitivo (Díaz, 2013).

Para entender la gentrificación en un espectro más amplio, es bueno saber que se puede hablar de la gentrificación dentro de dos variables muy palpables, que son gentrificación planificada, que se desarrolla a partir de la colaboración de gobernantes e inversionistas de iniciativa pública o privada) y la otra es la gentrificación espontánea, que se desarrolla por la suma de iniciativas individuales de habitantes 
que deciden habitar y consumir en barrios no mejorados por el gobierno (Delgadillo, 2016). En caso de la zona sur de Monterrey, es posible identificar características.

Por un lado, destaca el crecimiento descontrolado de la mancha urbana que hizo que los nuevos barrios se trasladaran hacia las periferias $y$, por otro lado, los nuevos barrios invadieron a los ya existente, consumiendo de sus negocios locales (tortillerías, verdulerías, tiendas de conveniencia, por nombrar algunos), simplemente porque la línea que marca el cambio de barrio, implica a su vez variaciones generalmente al alza del precio.

Para una parte del mundo académico la gentrificación no produce desplazamiento $\mathrm{y}$, al contrario, crea las condiciones para la constitución de ciudades más integradas. En ese sentido la gentrificación ayuda a fomentar la sustitución de comunidades marginales por población activa y responsable (Blomley,2004), diluye la concentración de la pobreza en los centros de las ciudades y fortalece el tejido social en barrios desfavorecidos, además, fortalece los vínculos de servicios entre los que llegan y los que viven en dichos espacios, combinando la polarización e interdependencia entre el servicio requerido y la fuente de trabajo, lo que genera oportunidades laborales para los sectores pobres que viven en la zona (Sabatini y Cáceres, 2004).

En América Latina los estudios sobre los procesos de gentrificación son relativamente recientes pero muy diversos. Varias investigaciones parten de una definición "clásica" que indica que la gentrificación se caracteriza por su contenido de clase y el despojo de sus barrios a la población de menores ingresos, para destinarlos a los negocios inmobiliarios y al consumo de población de mayores ingresos (Delgadillo, 2016) (Figura 2).

2.2 La mancha urbana y su crecimiento acelerado

La expansión de la mancha urbana origina una estructura polinuclear en la organización urbana de la ciudad, lo que hace que los usos y costumbres de los habitantes de los barrios, se vayan modificando o incluso perdiendo.

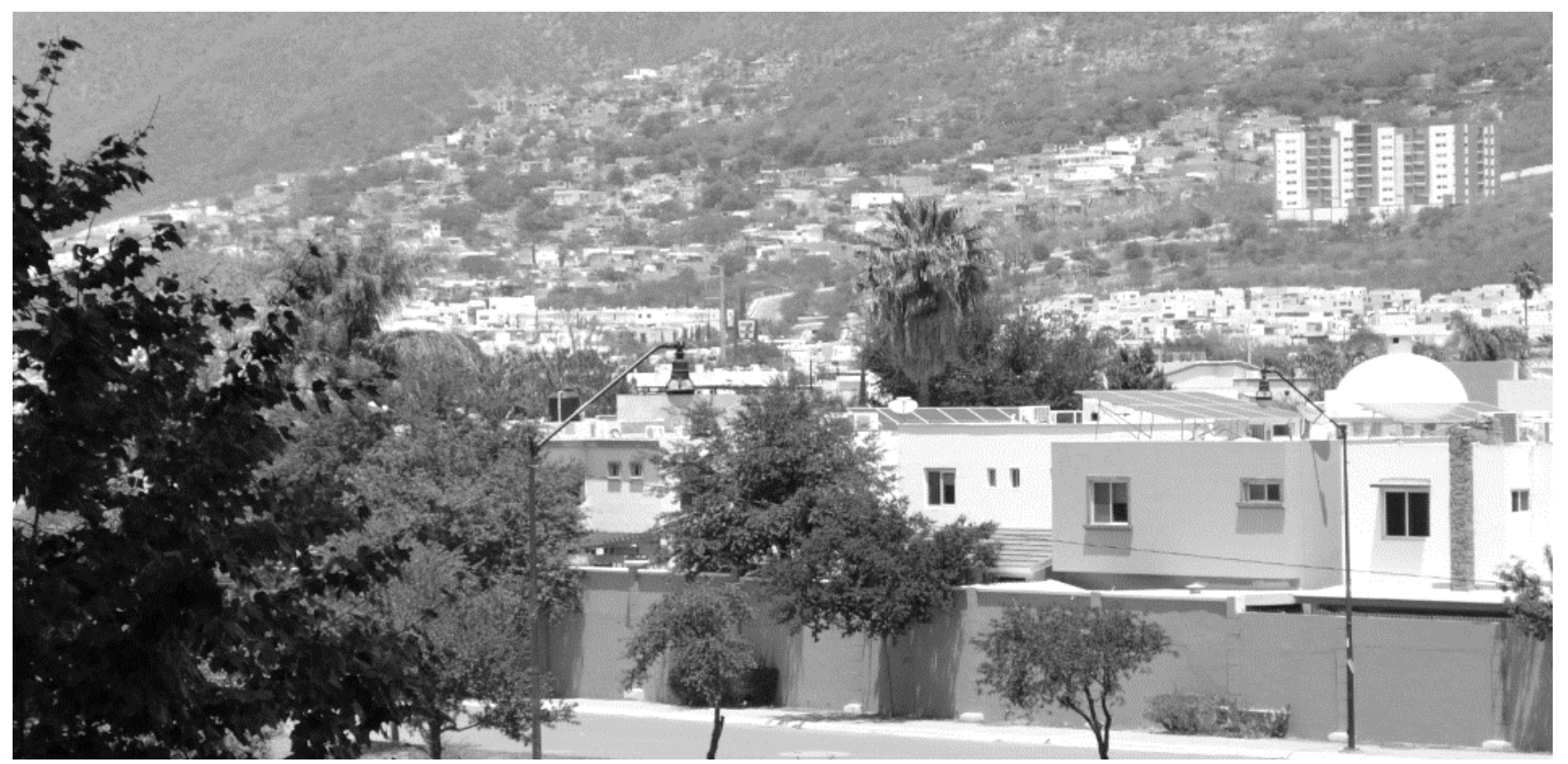

Figura 2: Contexto socioeconómico de la zona del Huajuco. Fuente: propia 


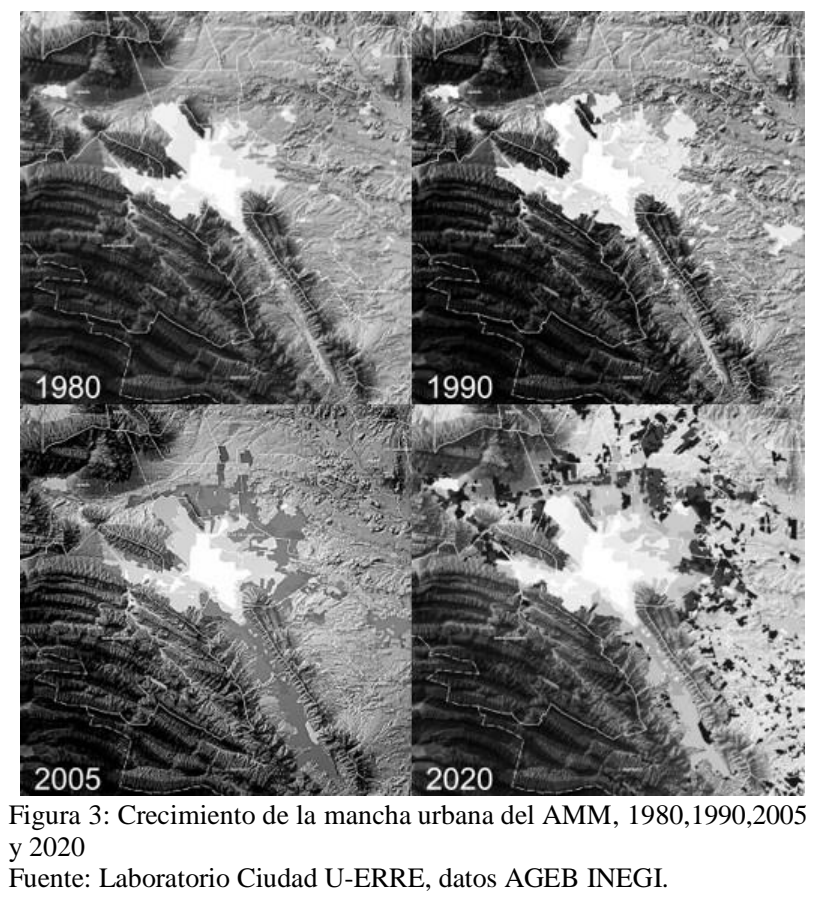

Es claro que el crecimiento en la zona se detonó por inmobiliarias comerciales que prometían una idea de vida tranquila lejos del bullicio de la zona metropolitana. A medida que ha pasado el tiempo, se ha visto esta zona muy invadida, pues los dichos comercios, crearon un boom de vivienda en esta zona, lo que ocasionó incrementos de la plusvalía en los barrios que antiguamente eran conocidos por ser de nivel socioeconómico medio y medio bajo. Esto, ha hecho que los mismos habitantes de la zona del Huajuco (Figura 2) por ejemplo, se sientan invadidos y ajenos a su contexto inmediato.

\subsection{Los desarrollos inmobiliarios}

El desarrollo inmobiliario es un proceso comercial en las grandes aglomeraciones urbanas que da paso a un patrón de asentamientos de alta densidad poblacional a uno de urbanización dispersa y más fragmentada. Este tipo de estructura territorial, denominada como de núcleos múltiples, parte de la reestructuración del uso del suelo urbano para hacer de otros sectores fuera del centro más importantes, (Aguilar, 2002) (Tabla 1). Asimismo, Moreno y Alvarado (2011) establecen que estos desarrollos se dan ante la importante demanda de viviendas y la falta de planeación presente en la mayoría de las ciudades del país, es necesario establecer nuevas formas de proyectar y construir viviendas, las cuales deberán tomar en cuenta los aspectos sociales, geográficos y ecológicos que permitan a las familias desarrollarse en un entorno adecuado y agradable.

Tabla 1. Desarrollos inmobiliarios de la zona del Huajuco

\begin{tabular}{lll}
\hline Nombre del proyecto & Tipo de uso & Año de inicio \\
\hline Plaza Villas Paseo & Comercial & 2017 \\
Esfera City Center & Comercial & 2015 \\
Pueblo Serena & Comercial & 2015 \\
Paseo la Rioja & Comercial & 2012 \\
Plaza Vía La Luz & Comercial & 2017 \\
Plaza Nacional 5500 & Comercial & 2016 \\
Soriana Hiper & Comercial & 2015 \\
HEB Valle Alto & Comercial & 2010 \\
Soriana La Estanzuela & Comercial & 2000 \\
Home Depot & Comercial Especializado & 2015 \\
Gilsa & Comercial Especializado & 2015 \\
Mercedes- Benz La Rioja & Comercial Especializado & 2020 \\
Torre Gaia & Residencial | vertical & 2019 \\
Frac. La Perla & Residencial & 2006 \\
Frac. Lomas del Vergel & Residencial & 2004 \\
Frac. Sierra Alta & Residencial & 2000 \\
Frac. Portal del Huajuco & Residencial & 2003 \\
La Herradura & Residencial & 2004 \\
Plaza valle alto 200 & Comercial & 2007 \\
\hline
\end{tabular}




\section{3.-MÉTODO}

Se diseño y aplicó una encuesta a 103 habitantes de la zona sur de Monterrey con una muestra no probabilística por bola de nieve, por conveniencia ya que los 103 habitantes en los que se realizó el muestreo no conforman el total de la población de la zona, específicamente en el área denominada Huajuco (así nombrada por el plan de desarrollo urbano de Monterrey 2013-2025). Esta zona ha mostrado una gran extensión de la mancha urbana, derivada de la llegada de nuevos desarrollos comerciales y habitacionales, donde se buscó conocer el perfil socioeconómico, densidad poblacional y su percepción ante los nuevos desarrollos.

Con esta investigación, se pretende demostrar el resultado mediático que ha tenido la expansión de la zona Sur en Monterrey, tomando como base de estudio los barrios de San ángel, Fomerrey-45, la condesa, ampliaciones nogales, granja postal, nueva Estanzuela, Ignacio Altamirano y los desarrollos inmobiliarios que han llegado a la zona, en los cuales se ha hecho el estudio de población considerando factores económicos, sociales, demográficos, etc. (Figura 2).

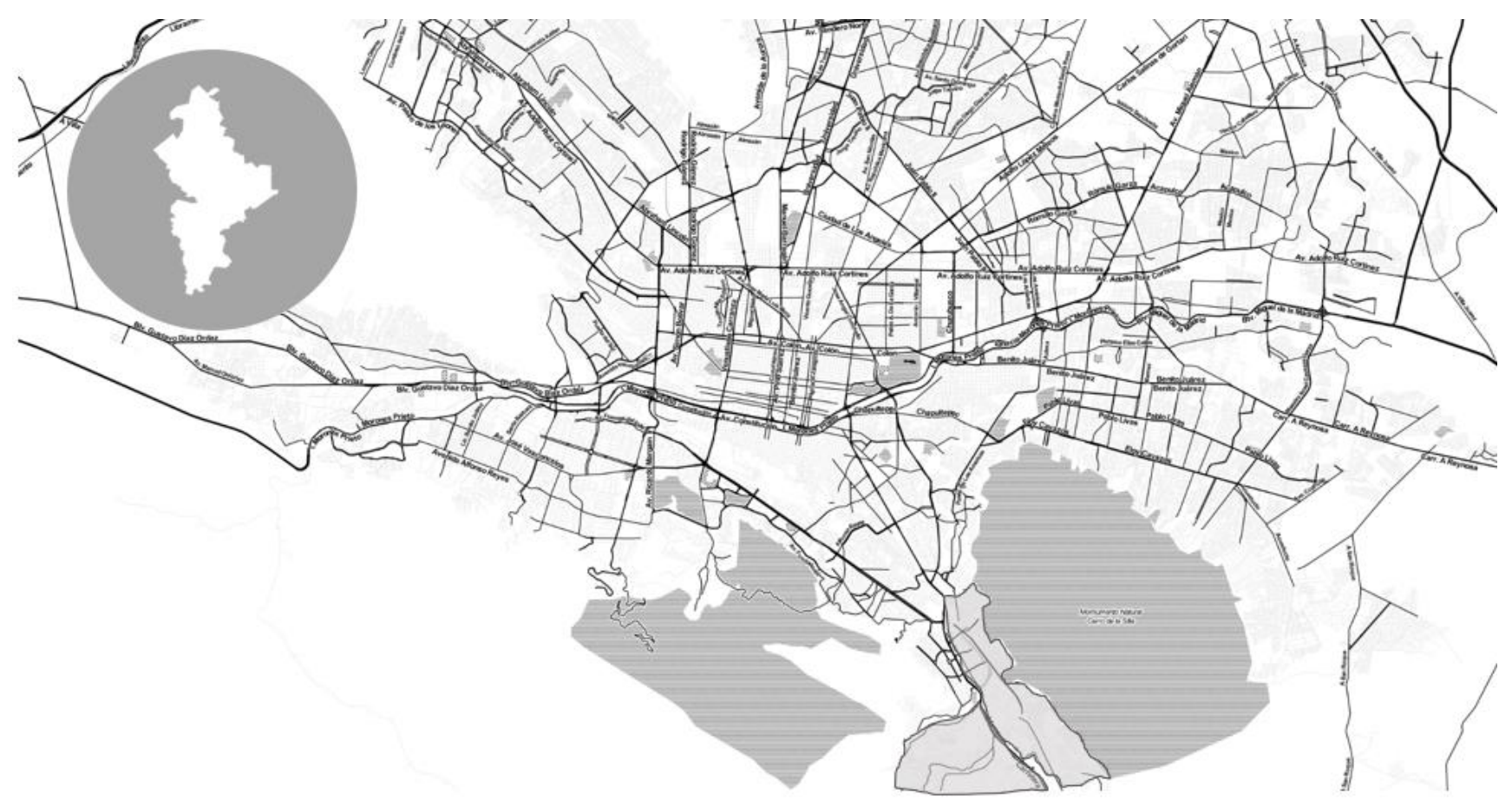

Figura 2: mapa de ubicación de la zona del Huajuco. Fuente: propia (Stamen design).

La encuesta se realizó por medio del método probabilístico sistemático con el programa de formularios de goolge, de manera virtual a través de una invitación a los habitantes para responder a 17 preguntas divididas en cuatro bloques; información general, información laboral, movilidad, vivienda y percepción del entorno. Esta encuesta se levantó en una semana. Otros datos consultados son el nivel de escolaridad, antigüedad en la vivienda y su percepción de seguridad y de invasión, para saber si realmente el habitante con más antigüedad se siente en armonía y satisfecho en términos de bienestar con su nuevo entorno. La base de datos fue vaciada en el programa IBM SPSS (paquete estadístico para las ciencias sociales) para la obtención de gráficos y tablas para una mejor comprensión de los resultados arrojados por la encuesta. 


\section{RESULTADOS}

Se realizo un estudio de la población en zona sur de Monterrey, para conocer el perfil socioeconómico, nivel de escolaridad, edad, género, movilidad, ingresos, vivienda y seguridad en su entorno. (tabla $2)$. s. la encuesta indica que el $63.11 \%$ se encuentra soltero. Dentro de los resultados sobre la percepción a sentirse invadidos un $63.11 \%$ se siente invadido sin embargo un $72.4 \%$ acude a centros comerciales de la zona mostrando un $77.3 \%$ comodidad al visitarlos.

Tabla 2 descripción de variables

\begin{tabular}{ll}
\hline Variable & Descripción \\
\hline Edad & Edad de los habitantes de la zona \\
Género & Identificación del género de los habitantes. \\
Estado civil & Situación de la persona para determinar su relación familiar. \\
Estudios & Nivel máximo de estudios de las personas encuestadas. \\
Situación laboral & Situación actual de trabajo del encuestado. \\
Ingresos & Percepción económica del habitante. \\
Movilidad & Medio de transporte de los habitantes de la zona \\
Antigüedad & Cantidad de años del habitante en el domicilio \\
Vivienda & Situación del habitante con relación al lugar donde habita. \\
Seguridad & Que tan seguro se siente el habitante del lugar donde vive. \\
Cambio & Cambio de domicilio actual \\
Crecimiento & Percepción del crecimiento de la zona \\
Invadido & Percepción de invasión por nuevos desarrollos inmobiliarios \\
Nuevos comercios & Percepción de los nuevos comercios en la zona \\
Acudir & Visita a centros comerciales \\
Comodidad & Nivel de comodidad al acudir a los centros comerciales. \\
\hline
\end{tabular}

Fuente: Elaboración propia.

La percepción del antiguo habitante de la zona es de no invasión respecto de los nuevos asentamientos (Figura 1). Lo anterior se debe a la adaptación de los

Figura 1 percepción de invasión de los habitantes de la zona de estudio 2021

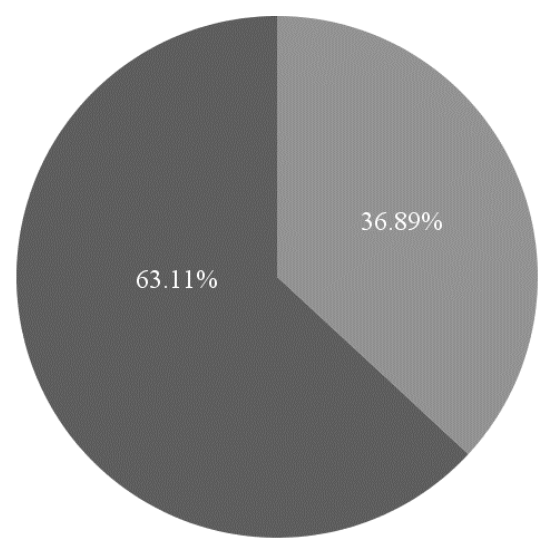

Invadido $\mathrm{Si}$
$\mathrm{N} \mathrm{No}$ habitantes a los nuevos comercios y desarrollos inmobiliarios. (Figura 3)

Un $35 \%$ de los encuestados tiene menos de cinco años de antigüedad mientras que un $22.3 \%$ tiene entre 20 y 30 años de residir en su domicilio. Esto quiere decir que actualmente hay mucha población joven la edad promedio es de 31 años. En el tema de la permanencia y el desplazamiento del habitante el $32 \%$ prefiere cambiar de domicilio y un $64 \%$ se quedaría en el lugar en donde vive mientras el $4 \%$ restante no contesto, con esto se confirma que al menos en esta zona de Monterrey la gentrificación no genera desplazamiento, sino al contrario está creando espacios que pueden ser utilizados por los habitantes de los barrios medio-bajos, y que también buscan mejorar el entorno para dejar atrás esa imagen de inseguridad que refleja el entorno. 


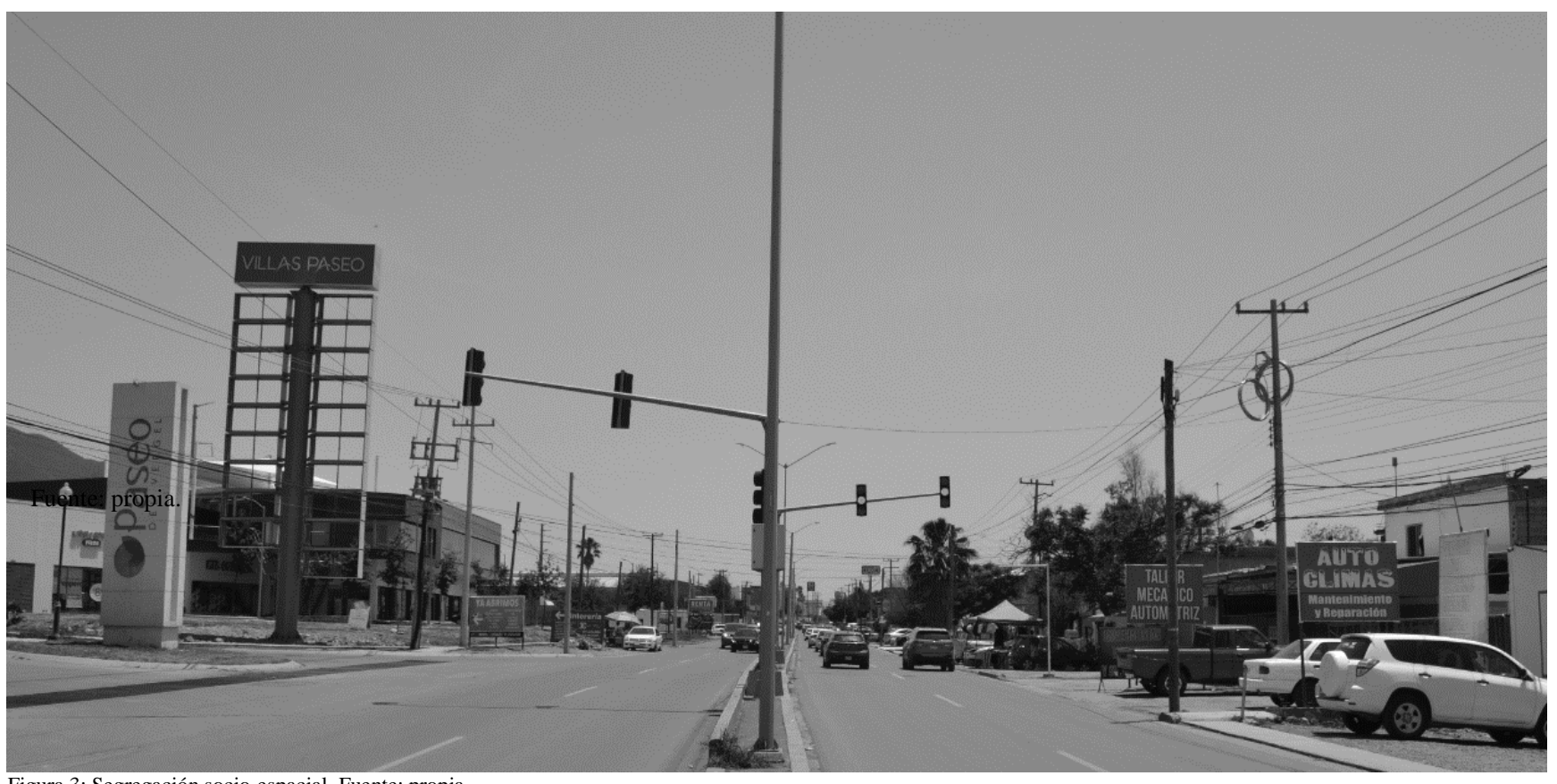

Figura 3: Segregación socio-espacial. Fuente: propia

La encuesta reveló que el $63.1 \%$ ha concluido sus estudios a nivel de licenciatura datos que reflejan la búsqueda de una mejora en la calidad de vida. Seguido de un $13.5 \%$ cuenta con maestría. (Figura 2).

Figura 2. Nivel de estudios

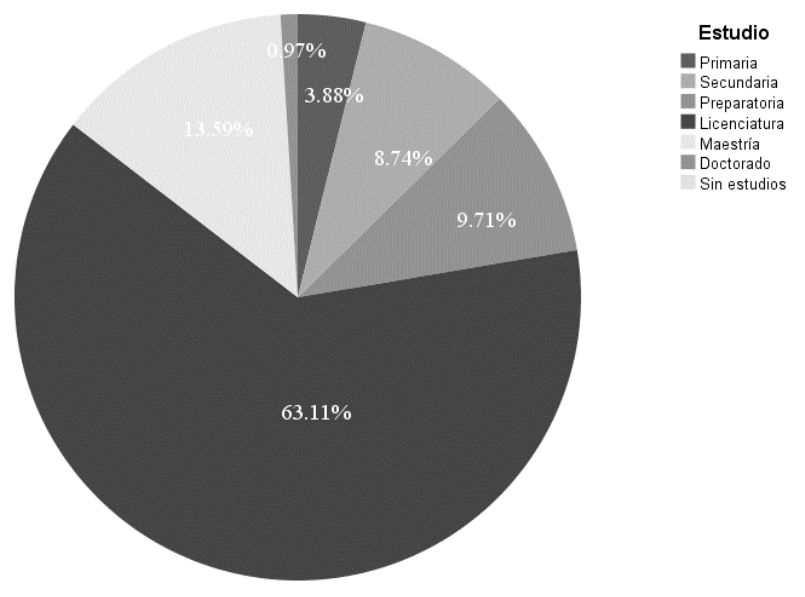

A manera de hipótesis es claro que el crecimiento de la zona detono un incremento comercial y habitacional que aparenta ser inclusivo, pero es notorio que estos desarrollos se inclinan para un nivel socioeconómico medio-alto, estas zonas son conocidas por ser de un nivel medio-bajo, pero el habitante de los barrios se siente privilegiado por contar con nuevos desarrollos cerca de su entorno ya que esto genera una mejor plusvalía y mejores lugares a los cuales acudir ya que un $80.6 \%$ se siente cómodo, un $6.8 \%$ se siente incómodo y solo el $1 \%$ menciono sentirse discriminado, mientras el $11.7 \%$ se siente observado al visitar los centros comerciales. Lo anterior describe que el habitante-invasor crea una tolerancia y convivencia sin distinción de clases social.

En relación al tipo de vivienda $65 \%$ cuenta con casa propia, un $19.4 \%$ rentada, un $12.6 \%$ prestada y un $2.9 \%$ tiene la casa intestada o en litigio. En el tema de seguridad un $29.1 \%$ se siente seguro siempre, mientras que un $32 \%$ solo en algunas ocasiones, otro $32 \%$ casi siempre se siente seguro y solo un $6.8 \%$ contesto que nunca. Con esto la percepción de seguridad indica que el habitante se siente tranquilo del barrio donde habita. (Tabla 3).

Fuente: Elaboración propia. 
Tabla 3. Variables sociodemográficas de los encuestados y porcentajes

\begin{tabular}{|c|c|c|}
\hline Concepto & Frecuencia & Porcentaje \\
\hline \multicolumn{3}{|l|}{ Género } \\
\hline Masculino & 56 & 54.4 \\
\hline Femenino & 47 & 45.6 \\
\hline Total & 103 & 100.0 \\
\hline \multicolumn{3}{|l|}{ Vivienda } \\
\hline Rentada & 20 & 19.4 \\
\hline Prestada & 13 & 12.6 \\
\hline Propia & 67 & 65.0 \\
\hline Intestada o en litigio & 3 & 2.9 \\
\hline Total & 103 & 100.0 \\
\hline \multicolumn{3}{|l|}{ Antigüedad } \\
\hline Menos de 10 años & 51 & 49.6 \\
\hline 10 a 20 años & 22 & 21.3 \\
\hline 20 a 30 años & 23 & 22.3 \\
\hline Más de 30 años & 7 & 6.8 \\
\hline Total & 103 & 100.0 \\
\hline \multicolumn{3}{|l|}{ Crecimiento } \\
\hline Más tráfico & 41 & 39.8 \\
\hline Más comercio & 32 & 31.1 \\
\hline Reducción de áreas verdes & 30 & 29.1 \\
\hline Total & 103 & 100.0 \\
\hline \multicolumn{3}{|l|}{ Seguridad } \\
\hline Siempre & 30 & 29.1 \\
\hline Casi siempre & 33 & 32.0 \\
\hline A veces & 33 & 32.0 \\
\hline Nunca & 7 & 6.8 \\
\hline Total & 103 & 100.0 \\
\hline \multicolumn{3}{|l|}{ Cambio } \\
\hline $\mathrm{Si}$ & 35 & 32.0 \\
\hline No & 68 & 64.1 \\
\hline Total & 103 & 100.0 \\
\hline \multicolumn{3}{|l|}{ Comodidad } \\
\hline Discriminado & 1 & 1.0 \\
\hline Observado & 12 & 11.7 \\
\hline Cómodo & 83 & 80.6 \\
\hline Incómodo & 7 & 6.8 \\
\hline Total & 103 & 100.0 \\
\hline
\end{tabular}

Fuente: Elaboración propia. 


\section{CONCLUSIONES}

En este artículo se estudiaron diferentes variables para conocer el perfil del habitante y su percepción sobre, el proceso de gentrificación que se está llevando a cabo en la zona debido a la llegada de los nuevos desarrollos inmobiliarios que, visualmente es notoria la segregación de los barrios ya existentes, sin embargo, los resultados indicaron que se ha creado una sinergia entre los barrios de perfil mediobajo y los nuevos desarrollos comerciales y habitacionales.

Los habitantes de la zona acostumbran a acudir a estos nuevos comercios y se sienten cómodos, pero es evidente que aún se muestran inconformes por que la llegada de los nuevos habitantes es perjudicial para la movilidad.

La percepción de sentirse invadido por parte del habitante es notoria y se ha elevado. La mancha urbana a operado en detrimento del bienestar de la población en términos de percepción de tranquilidad respecto de años anteriores. Este crecimiento ocasiona densificación a través del aprovechamiento del uso de suelo. Los desarrollos inmobiliarios crean espacios de uso mixto; comercio, vivienda, oficinas y proyectos de vivienda de tipo vertical, principal característica de los desarrollos sitiados en la zona sur de Monterrey y las demás periferias urbanas. Estas zonas se están convirtiendo en subcentros poblacionales, en un entorno con la cobertura tanto de los servicios básicos como de esparcimiento, y de las actividades que prescriben el desarrollo de la ciudad.

El tránsito es uno de los temas de mayor problemática debido a que las vialidades no están diseñadas para semejante crecimiento. De esta manera, la densificación de la población ocasiona que vías internas que, anteriormente no eran conocidas, sean ahora de uso frecuente de los nuevos habitantes.

Es evidente que se lleve a cabo una planificación adecuada de los espacios porque la creación de los desarrollos inmobiliarios se realizó sin estudios previos de movilidad.

Los comercios de la zona se han visto beneficiados. La seguridad que percibe el habitante es, lo que rescribe una mejora en la perspectiva de los habitantes en este rubro.

Este análisis permite concluir que la gentrificación no produce desplazamiento de los habitantes, sino al contrario ha creado espacios para la convivencia de diferentes perfiles socioeconómicos. Por ello, el ambiente sin distinción social acompañado de un mejor dinamismo en términos económicos de los barrios desfavorecidos debido a la creación de nuevos empleos, además de la creación de espacios recreativos para todos los sectores de la zona. 


\section{REFERENCIAS}

Aguilar A, (2002). Las megaciudades y las periferias expandidas. Ampliando el concepto en Ciudad de México. EURE, 28(85), 121-149.

Alanís F, Glafiro J. \& Cervantes J. (1995), Atlas de Monterrey, Instituto de Estudios Urbanos de Nuevo León, El Colegio de México.

Aparicio C., Ortega M., \& Sandoval E. (2011). La segregación socio-espacial en Monterrey a lo largo de su proceso de metropolización. Scienfic Electronic Library Online, 52, 35.

Barbarín J. (1995) "Geología y Geomorfología” Atlas de Monterrey., Instituto de Estudios Urbanos de Nuevo León, El Colegio de México.

Casgrain A. \& Janoschka M. (mayo-agosto, 2013). Gentrificación y resistencia en las ciudades latinoamericanas El ejemplo de Santiago de Chile. Scienfic Electronic Library Online, 10, 26. abril 2021, De Andamios.

Díaz I. (2013). La gentrificación en la cambiante estructura socio-espacial de la ciudad (1030). Instituto de geografía, Universidad Autónoma de México.

Delgadillo V. (noviembre 2016). Ciudad de México, quince años de desarrollo urbano intensivo: la gentrificación percibida. Scienfic Electronic Library Online, 31(88): 101-129, 29. abril 2021, De Universidad Autónoma de la Ciudad de México

Mendoza F. (2016). La gentrificación en los estudios urbanos: una exploración sobre la producción académica de las ciudades. Scienfic Electronic Library Online, 18, 24. abril 2021, De Cadernos Metrópole.

Moreno, R. y Alvarado, E. (2011). El entorno social y su impacto en el precio de la vivienda: un análisis de precios hedónicos en el área metropolitana de Monterrey. Trayectorias: Revista de Ciencias Sociales. Año 14, Núm. 33, 131-147.

Sabatini, F. \& Cáceres, G. (2004). "Los barrios cerrados y la ruptura del patrón tradicional de segregación en las ciudades latinoamericanas: el caso de Santiago de Chile". Scienfic Electronic Library Online. 